\title{
Stagnation-point flow towards a vertical plate embedded in a porous medium
}

\begin{abstract}
The steady stagnation-point flow towards a heated vertical surface embedded in a porous medium is investigated. The governing partial differential equations are reduced to a system of nonlinear ordinary differential equations using a similarity transformation. The shooting method is used to solve the similarity equations for different values of the mixed convection parameter. The features of the flow and heat transfer characteristics are analyzed and discussed. It is found that dual solutions exist for both assisting and opposing flows. The skin friction coefficient and the local Nusselt number increase as the mixed convection parameter increases.
\end{abstract}

Keyword: Axisymmetric flow; Mixed convection; Plane flow; Porous medium 